\title{
AIT Inversion and Site Applications Using Various Forward Algorithms
}

\author{
Zhenhua Liu ${ }^{a}$ and Jianbo Dai ${ }^{b}$ \\ Mechanical Engineering College, Xi'an Shiyou University, Xi'an, 710065, China \\ aliuzhenhua@xsyu.edu.cn, bd jianbo@foxmail.com
}

Keywords: Array induction tool; Logging; Inversion; Resistivity; Forward algorithms

\begin{abstract}
An inversion algorithms was suggested for array induction logging curves. Three parameters, true-formation resistivity, invasion depth and invasion-zone resistivity, were obtained simultaneously from the present inversion calculation using various forward algorithms. The inversed algorithm using numerical mode-matching method as forward calculation can yield stable and satisfied results. The inversion using geometry factor theory as forward calculation can save more computer time. Synthetic formation models and site applications illustrated the efficiency of the present algorithm.
\end{abstract}

\section{Introduction}

Saturation of hydrocarbon in a reservoir is an important parameter during oil exploration; but it can not be measured directly in site and it relates to formation resistivity [1]. Formation resistivity is usually determined from resistivity logging devices such as traditional dual-induction tool that can only provide two responses with medium- and deep-detective depths [1]; or new array induction tool [2], which can record five logging data with five investigating depths simultaneously [3-5].

However, the resistivity measurement was influenced by environmental effects such as borehole, mud, invasion, shoulder bed, and so on. Hence, the resistivity logging data would deviate from the true-formation resistivity. Inversion technique is an efficient method to eliminate environmental influence as far as possible and obtain the true-formation resistivity from logging data.

AIT measurement is influenced by environmental effects also, so that the logging responses deviate from the true-formation resistivity. In order to eliminate the environmental influence and obtain the true-formation resistivity, a fast inversion technique was suggested in the present study. Since the inversion calculation was dependent on the forward algorithm, two forward calculation algorithms, geometry factor (GF) theory [1] and numerical mode-matching (NMM) method[6-8], were used in the inversion calculation respectively.

\section{Forward Algorithms for AIT Response}

A two-dimensional axisymmetric formation model was considered in the present calculation. In the direction of vertical to the ground, the formation was divided into $N$ layers. After the porous formation was opened, the drilling-mud filtrate pours. The native fluids, water and hydrocarbon, were displaced by the filtrate under the pressure differential between borehole and formation. Thus, an invasion zone, with radius $r_{h}$ and mud resistivity $R_{m}$, formed near to the borehole. The present model included borehole with radius $r_{h}$ and mud resistivity $R_{m}$, formation with thickness $H$ and true-formation resistivity $R_{t}$. There was a shoulder with resistivity $R_{s}$ between reservoirs.

AIT apparent responses $R_{a}$ can be obtained from geometry factor theory:

$$
\frac{1}{R_{a}}=\operatorname{Re} \sum_{j=1}^{n} \sum_{k=1}^{m} \frac{w_{k j}}{R_{a}^{(j)}\left(z-z_{k j}\right)}
$$

Where $R_{a}^{(j)}$ is apparent resistivity of $j$-th channel of AIT coils; $w_{k j}$ is weight factor; $n$ and $m$ are the number of receive and transmit coils respectively. The $z$ axis is along the direction of device length. 
Another method to calculate AIT responses is NMM method [6-8]. The numerical analysis was used in the radial direction and analysis method was adopted in the vertical direction. It transfers the original two-dimensional problem into a one-dimensional eigenvalue problem and layered medium problem, which can be modeled efficiently by a recursion procedure.

\section{Inversion of AIT Logging Data}

AIT measurement provides five logging curves with five detective depths in radial direction. When drilling mud filtrate invade into a reservoir, invasion zone formed. The invasion zone was usually described using invasion depth $r_{i}$ and resistivity $R_{x o}$, both of them were unknown. Thus, three parameters, $r_{i}, R_{x o}$, and true-formation resistivity $R_{t}$ need to be determined from inversion algorithm.

In general problem for resistivity logging inversion, a forward model with $m$ parameters is used to fit the logging data with $n$ data. It can be written as following:

$$
R_{n}=F_{n}\left(R_{t}, R_{x o}, r_{i}, S_{n}\right) \quad n=1,2, \ldots, 5
$$

Where $R_{n}$ is logging data. $R_{1}, R_{2}, R_{3}, R_{4}$, and $R_{5}$ denote the apparent resistivities of AIT measurement with five investigating depths respectively. $R_{t}, R_{x o}$ and $r_{i}$ are the inversed parameters. $S_{n}$ means other parameter in the forward model. Eq.(2) is a nonlinear equation. If the initial guesses of $R_{t}$, $R_{x o}$ and $r_{i}$ were given, it can be linearized according to the method of solving least-squares problems $[9,10]:$

$$
R_{n}=R_{n}^{0}+\frac{\partial F_{n}}{\partial R_{t}} \delta R_{t}+\frac{\partial F_{n}}{\partial R_{x o}} \delta R_{x o}+\frac{\partial F_{n}}{\partial r_{i}} \delta r_{i} \quad n=1,2, \ldots, 5
$$

This equation can be rewritten as a new difference vector:

$$
\vec{\varepsilon}=\vec{R}-\vec{R}^{0}=J \Delta \vec{P}
$$

Where, $\vec{R}=\left(R_{1}, R_{2}, R_{3}, R_{4}, R_{5}\right)^{T}$ is the vector of logging data;

$\vec{R}^{0}=\left(R_{1}^{0}, R_{2}^{0}, R_{3}^{0}, R_{4}^{0}, R_{5}^{0}\right)^{T}$ is the guessed data vector in forward model; $\vec{P}=\left(R_{t}, R_{t}, r_{i}\right)^{T}$ is the vector of inversed parameters and $J$ is Jacobi matrix.

Eq.(3) is a linear equation system related to modified step $\Delta \vec{P}$. After its damping least square solution is obtained, a new vector of model parameters is generated. This new parameter is input into forward model to generate new guess data. Thus, new Jacobi matrix $J$ and new difference vector in Eq.(3) are obtained. They are used to generate new increment $\Delta \vec{P}$ and new Jacobi matrix. The process is repeated until the expectant results that satisfy iteration error is reached.

\section{Synthetic Data Inversion}

A synthetic formation model was constructed to check the present AIT inversion algorithm. In this model, the true-formation resistivity $R_{t}$ was set to be $20.0 \Omega \mathrm{m}$, the invasion resistivity $R_{x o}$ was $5 \Omega \mathrm{m}$, and the invasion depth $r_{i}$ was $1.016 \mathrm{~m}$. The resistivity of shoulder bed $R_{s}=2.0 \Omega \mathrm{m}$. The reservoir thickness $H$ was chosen to be $10,7,5,4,3,2.7,2.4,2.1,1.8,1.5,1.2,0.9,0.6,0.45$, and $0.3 \mathrm{~m}$ respectively in order to address the vertical resolution of AIT measurement. Fig. 1(a) illustrated the apparent resistivity $R_{a}$ of AIT. The vertical direction denotes the depth from the surface of ground. AIT10, AIT20, AIT30, AIT60 and AIT90 usually denote the five arrays with the various investigation depths of 10, 20, 30, 60 and 90inch $(0.25,0.51,0.76,1.52$ and 2.29) respectively. Figure $1(\mathrm{~b}) \sim(\mathrm{g})$ were the true values (dash lines) and inversed results (solid lines) for $R_{t}, R_{x o}$ and $r_{i}$. Both the geometry factor (GF) theory and numerical mode-matching (NMM) method were used as forward algorithms during the inversion calculation. They were marked by GF and NMM in Fig. 1 respectively. 
In the present synthetic formation model, the invasion resistivity $R_{x o}, 5 \Omega \mathrm{m}$, was less than the true-formation resistivity $R_{t}, 20.0 \Omega \mathrm{m}$. Thus, the resistivity in the vicinity of borehole was low. It affected the shallow investigating AIT arrays, such as AIT10 and AIT20; so that they obtained small apparent resistivities. Whereas the deep investigating AIT arrays, such as AIT60 and AIT90, can still record initial formation information of high resistivity. Therefore, the forward calculations both of GF and NMM method indicated that the apparent resistivities of deep investigating AIT arrays was great than the values of shallow investigating AIT arrays, as shown in Fig. 1(a).

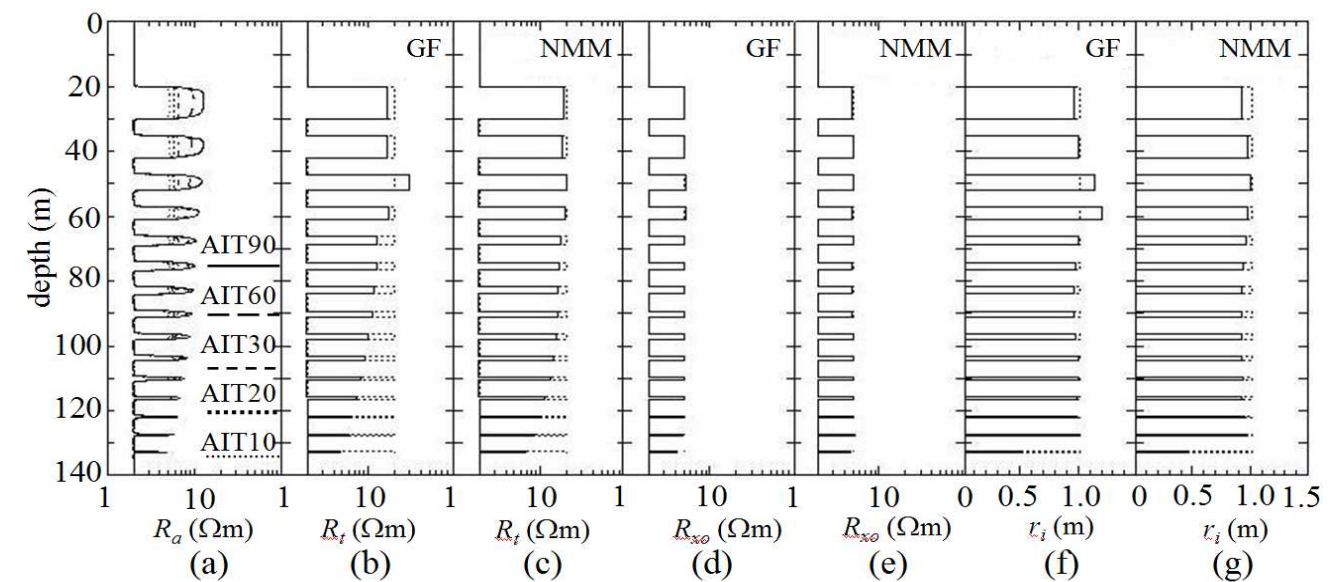

Figure 1. Finite inversion results for a synthetic formation: the dash lines and solid lines in (b) (g) were the true values and inversed results using GF or NMM as forward algorithm respectively.

Fig. 1 also illustrated that the inversed results using NMM as forward calculation can yield stable and satisfied results comparing with GF theory, especially for inversed $R_{t}$ and $r_{i}$. However, the inversed algorithm using geometry factor theory as forward calculation can save more computer time.

\section{Applications in Site}

Fig. 2 illustrated two examples of applications in an oil field located at North China. The curves in left boxes were AIT logging curves in the interval 530 620m for well A in Fig. 2(a) and 1980 2200 for well B in Fig. 2(b) respectively.

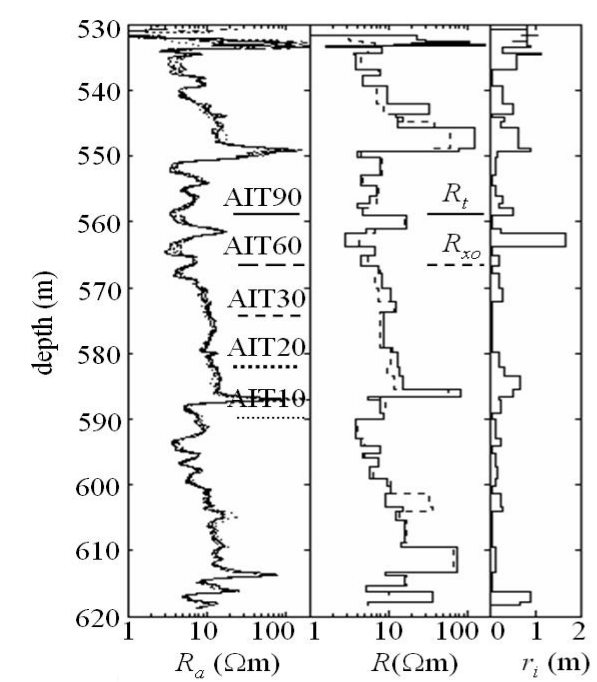

(a) well A: the interval 530 620m

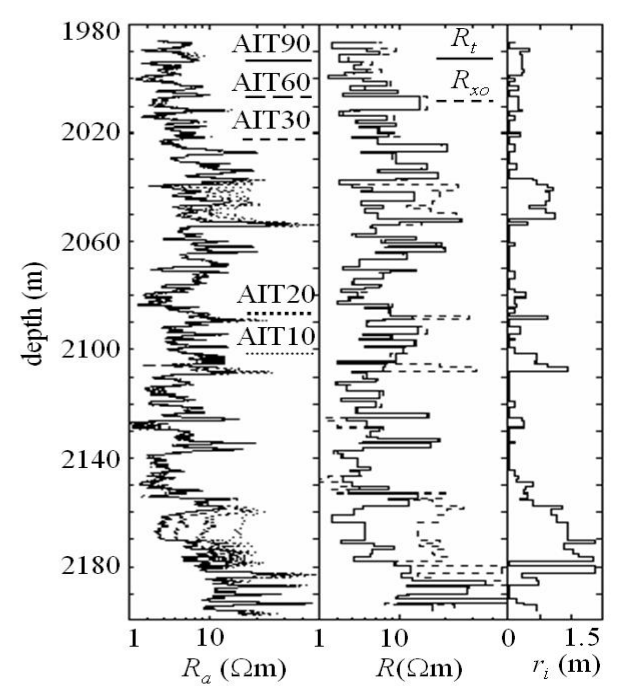

(b) well B: the interval 1800 2220m

Figure 2. Finite AIT logging curves and inversion results 
In Fig. 2(a) and Fig. 2(b), the curves in middle boxes were inversed true-formation resistivity $R_{t}$ and inversed invasion-zone resistivity $R_{x o}$; the curves in the right box were inversed invasion depth $r_{i}$. The numerical mode-matching method was used in forward calculation for the present applications in order to reach accurate inversion results.

In Fig. 2a, the inversed $R_{x o}$ was less than the inversed $R_{t}$; hence, the apparent resistivities of shallow detective AIT arrays were less than the logging data of the arrays with deep investigation depth for most layers. In Fig. 2b, for most layers, $R_{x o}>R_{t}$; hence, shallow detective readings were great than the deep detective measurements. The present inversion model can generate convergent results for both cases. When the invasion depth $r_{i}$ got large value, it means the invasion zone extent to the deep formation, so that the invasion effects on logging became heavily. The logging readings deviated from the true-formation resistivity obviously. The inversion algorithm was a reasonable method to eliminate the environmental influences on the measurement for true-formation resistivity.

\section{Conclusions}

The array induction tool not only modified the resolution in vertical direction, but also it provides five logging curves that correspond to five detective depths in radial direction. They were enough to inverse the true-formation resistivity, invasion depth and resistivity in invasion zone.

Forward algorithm is important during inversion calculation. Although the inversion algorithm using NMM as forward calculation can yield stable and satisfied results, the inversion using GF theory as forward calculation can save more computer time.

The present inversion results can used to explain the departures of five AIT logging curves and to correct the resistivity deviation from the true value due to the influence of environment effects. It is helpful for log analysts during logging interpretation.

\section{Acknowledgements}

The financial support for this work provided by the Special Research Project in Shaanxi Province Department of Education(15JK1562) is gratefully acknowledged.

\section{References}

[1] Tittman J, Geophysical Well Logging (Academic Press Inc., U.S.,1986)

[2] T. Barber, A. Orban, G. Hazen, T. Long, A multiarray induction tool optimized for efficient wellsite operation, SPE annual technical conference and exhibition: formation evaluation and reservoir geology (Dallas TX, U.S., Oct.22-25, 1995), p.549-561

[3] J. S. Hou, and B. Michael, Correction for borehole effect of multi- component array induction log data, Progress in Electromagnetics Research Symposium (Cambridge, July 5-8, 2010)

[4] B. Corley, R. Khokhar, H. M. Maurer, et.al., A study of differences in array induction and multi-laterolog responses in a well drilled with high-salinity water-based mud, SPE Oil and Gas India Conference and Exhibition (Mumbai, India, March 28-30, 2012)

[5] L. S. Martin, J. Buchman, M. Bittar M, et. al., Application of New Asymmetrical Array Induction Tool In Hostile Environments, 48th Annual Logging Symposium (Austin, U.S., June 3-6, 2007)

[6] W. C. Chew, Z. P. Nie, Q. H. Liu, et.al., An efficient solution of electrical well logging tools in a complex environment, IEEE Trans. Geosci. Remote Sensing, Vol.29(1991)No.2, p.308-313

[7] Mahmood-ul-Hassan, M.H. Meylan and M. Sumbul, Mode matching analysis for wave scattering in triple and pentafurcated spaced ducts, Mathematical Methods in the Applied Sciences, Vol.39(2016) No.11, p.3043-3057

[8] R. Dey, S. Chakrabarty, R. Jyoti and T. Kurian, Synthesis and analysis of multi-mode profile horn using mode matching technique and evolutionary algorithm, IET Microwaves, Antennas \& Propagation, Vol.10(2016)No.3, p.276-282 
[9] M. Arioli and S. Gratton, Linear regression models, least-squares problems, normal equations, and stopping criteria, Computer Physics Communications, Vol.183(2012)No.11, p.2322-2336

[10]Z. Lin, D. Xiao, F. Fang, C. C. Pain and I. M. Navon, Non-intrusive reduced order modelling with least squares fitting, Inter. J. Num. Meth. in Fluids, Vol.83(2017)No.3, p.291-306 